\title{
Big-bang nucleosynthesis and leptogenesis in the CMSSM
}

\author{
Munehiro Kubo, ${ }^{1, *}$ Joe Sato, ${ }^{1, \dagger}$ Takashi Shimomura, ${ }^{2, \$}$ Yasutaka Takanishi, ${ }^{1, \S}$ and Masato Yamanaka ${ }^{3, \|}$ \\ ${ }^{1}$ Department of Physics, Saitama University, Shimo-Okubo 255, 338-8570 Saitama Sakura-ku, Japan \\ ${ }^{2}$ Faculty of Education, University of Miyazaki, Gakuen-Kibanadai-Nishi 1-1, 889-2192 Miyazaki, Japan \\ ${ }^{3}$ Maskawa Institute, Kyoto Sangyo University, Kyoto 603-8555, Japan
}

(Received 21 March 2018; published 8 June 2018)

\begin{abstract}
We have studied the constrained minimal supersymmetric standard model with three right-handed neutrinos, and investigated whether there still is a parameter region consistent with all experimental data/ limits such as the baryon asymmetry of the Universe, the dark matter abundance and the lithium primordial abundance. Using Casas-Ibarra parametrization, we have found a very narrow parameter space of the complex orthogonal matrix elements where the lightest slepton can have a long lifetime, which is necessary for solving the lithium problem. We have studied three cases of the right-handed neutrino mass ratio (i) $M_{2}=2 \times M_{1}$, (ii) $M_{2}=4 \times M_{1}$, (iii) $M_{2}=10 \times M_{1}$, while $M_{3}=40 \times M_{1}$ is fixed. We have obtained the mass range of the lightest right-handed neutrino that lies between $10^{9}$ and $10^{11} \mathrm{GeV}$. The important result is that its upper limit is derived by solving the lithium problem and the lower limit comes from leptogenesis. Lepton flavor violating decays such as $\mu \rightarrow e \gamma$ in our scenario are in the reach of MEG-II and Mu3e.
\end{abstract}

DOI: $10.1103 /$ PhysRevD.97.115013

\section{INTRODUCTION}

The standard models (SMs) of particle physics and cosmology have been successful at understanding most of the experimental and observational results obtained so far. Nonetheless, there are several phenomena which cannot be explained by these models. Among such phenomena, the mass and mixing of neutrinos, the baryon asymmetry of the Universe (BAU), the existence of the dark matter (DM), and the so-called lithium (Li) problems are compelling evidence that new physics laws are required. If all of these phenomena are addressed in particle physics, the new physics laws should be incorporated in a unified picture beyond the SM of particle physics.

Neutrino oscillation experiments (see Ref. [1] for recent review and global fit analysis) and cosmological observations $[2,3]$ revealed that the masses of neutrinos are much lighter than those of other known SM particles. To generate such tiny masses, many mechanisms have been proposed, among which the most well-studied and simplest is the

\footnotetext{
*kubo@krishna.th.phy.saitama-u.ac.jp

†joe@phy.saitama-u.ac.jp

*shimomura@cc.miyazaki-u.ac.jp

\$yasutaka@krishna.th.phy.saitama-u.ac.jp

masato.yamanaka@cc.kyoto-su.ac.jp
}

Published by the American Physical Society under the terms of the Creative Commons Attribution 4.0 International license. Further distribution of this work must maintain attribution to the author(s) and the published article's title, journal citation, and DOI. Funded by SCOAP.
type-I seesaw mechanism [4-8]. In this mechanism, the heavy Majorana right-handed (RH) neutrinos are introduced and, thus, the Yukawa interactions of left-handed (LH) and RH neutrinos can be formed with the Higgs scalar, which gives rise to the flavor mixings in the neutrino sector. After integrating out the RH neutrinos, the LH neutrino masses become very light due to the suppression factor which is proportional to the inverse of the Majorana mass scale. Thus, when we make use of the seesaw mechanism, we can successfully generate the phenomenologically required masses and mixings of $\mathrm{LH}$ neutrinos.

Furthermore, the seesaw mechanism has another virtue, generating the baryon asymmetry [2] through leptogenesis [9]. At the early stage of the Universe, the RH Majorana neutrinos are produced in the thermal bath. As the temperature decreases to their mass scale, these neutrinos go to out-of-thermal equilibrium, and at that time they decay into leptons with Higgs or antileptons with anti-Higgs. If $C P$ symmetry is violated in the neutrino Yukawa coupling, the decay rates into lepton and antilepton are obviously different. That means that the lepton number asymmetry is generated through the decays of the heavy Majorana RH neutrinos, and then the lepton number asymmetry is converted to the baryon asymmetry by the sphaleron process $[10,11]$ : the seesaw mechanism explains two phenomena simultaneously. (see, e.g., Refs. [12-18])

The existence of DM is also a problem [19]. The dark matter must be a massive and stable or a very long-lived particle compared with the age of the Universe and not 
carry electric or color charges. LH neutrino is the only possible candidate for the DM within the SM; however, this possibility already has been ruled out because neutrino masses are too light. Thus, one should extend the SM so that the DM is incorporated. Supersymmetry (SUSY) with $R$ parity is one of the attractive extensions in this regard, where the lightest SUSY particle (LSP) becomes absolutely stable. In many SUSY models, the LSP is the lightest neutralino that is a linear combination of neutral components of gauginos and Higgsinos that are SUSY partners of electroweak gauge bosons and the Higgses, respectively. Therefore, the lightest neutralino LSP is a good candidate for the DM, and in fact the abundance of the neutralino LSP can be consistent with observational ones of the DM [19] in specific parameter regions. In particular, the so-called coannihilation region is very interesting, in which the neutralino DM and the lighter stau, SUSY partner of the tau lepton, as the next-LSP (NLSP) are degenerate in mass [20]. When the mass difference of the neutralino LSP and the stau NLSP is smaller than $\mathcal{O}(100) \mathrm{MeV}$, the stau NLSP becomes long-lived so that it can survive during the bigbang nucleosynthesis (BBN) [21-23]. Thus, the existence of the stau NLSP affects the primordial abundance of light elements. One can expect to find evidence of the stau NLSP in the primordial abundance of the light elements.

It has been reported that there are disagreements on the primordial abundance of ${ }^{7} \mathrm{Li}$ and ${ }^{6} \mathrm{Li}$ between the standard BBN prediction and observations. The prediction of the ${ }^{7} \mathrm{Li}$ abundance is about 3 times larger than the observational one $(1.6 \pm 0.3) \times 10^{-10}$ [24-26]. This discrepancy hardly seems to be solved in the standard BBN with the measurement errors. This is called the ${ }^{7} \mathrm{Li}$ problem. The ${ }^{6} \mathrm{Li}$ abundance also disagrees with the observations. The predicted abundance is about $10^{3}$ smaller than the observational abundance ${ }^{6} \mathrm{Li} /{ }^{7} \mathrm{Li} \simeq 5 \times 10^{-2}$ [27]. Although this disagreement is less robust because of uncertainties in the observations, it is called the ${ }^{6} \mathrm{Li}$ problem.

Since the disagreements cannot be attributed to nuclear physics in the BBN [28], one needs to modify the standard $\mathrm{BBN}$ reactions. In Ref. [29], the authors have shown in the minimal SUSY standard model (MSSM) that negatively charged stau can form bound states with light nuclei and immediately destroy the nuclei through internal conversion processes during the BBN. Further, a detailed analysis [30] has shown that in the coannihilation region, where the lightest neutralino LSP is the DM and the stau NLSP has a lifetime of $\mathcal{O}\left(10^{3}\right)$ sec, $\mathrm{Li}$ and beryllium (Be) nuclei are effectively destroyed. The primordial abundance of ${ }^{7} \mathrm{Li}$ is reduced, while such a stau can promote the production of ${ }^{6} \mathrm{Li}$ [31]. It turns out that both densities become the observational values. This is a solution of the dark matter and the Li problems in the MSSM scenario. It should be noted that the SUSY spectrum is highly predictive in this parameter region. In Ref. [32], the authors also showed the whole SUSY spectrum in which the lightest neutralino mass is between 350 and $420 \mathrm{GeV}$ in the constrained MSSM (CMSSM). This result is consistent with nonobservation of SUSY particle at the LHC experiment so far. However, it is in the reach of the LHC Run-II.

In this article, we consider the CMSSM with the type I seesaw mechanism as a unified picture which successfully explains all phenomena as we have mentioned above. We aim to examine this model through searches of the longlived charged particles at the LHC and lepton flavor violation (see, e.g., Refs. [14,33-47]) at MEG-II, Mu3e and Belle-II experiments. This paper is organized as follows. In Sec. II, we review the CMSSM with the heavy RH Majorana neutrinos. In Sec. III, we show cosmological constraints such as dark matter, BBN and BAU, which we require to the model in our analysis. Then, we present the parameter sets of the CMSSM and RH Yukawa coupling which satisfies all requirements in Sec. IV. Predictions on lepton flavor violating decays are shown in Sec. V. The last section is devoted to a summary and discussion.

\section{MODEL AND NOTATION}

We consider the MSSM with RH Majorana neutrinos (MSSMRN). The superpotential for the lepton sector is given by

$\mathcal{W}_{l}=\hat{E}_{\alpha}^{c}\left(Y_{\mathrm{E}}\right)_{\alpha \beta} \hat{L}_{\beta} \cdot \hat{H}_{d}+\lambda_{\beta i} \hat{L}_{\beta} \cdot \hat{H}_{u} \hat{N}_{i}^{c}-\frac{1}{2}\left(M_{\mathrm{N}}\right)_{i j} \hat{N}_{i}^{c} \hat{N}_{j}^{c}$.

Here, $\hat{L}_{\alpha}$ and $\hat{E}_{\alpha}^{c}(\alpha=e, \mu, \tau ; i, j=1,2,3)$ are the chiral supermultiplets, respectively, of the $S U(2)_{L}$ doublet lepton and of the $S U(2)_{L}$ singlet charged lepton in the flavor basis which is given as the mass eigenstate of the charged lepton, that is, the eigenstate of $Y_{E}$ and hence implicitly $\left(Y_{E}\right)_{\alpha \beta}=$ $y_{\alpha} \delta_{\alpha \beta}$ is assumed. Similarly $\hat{N}_{i}^{c}(i=1,2,3)$ is that of the RH neutrino and the indices denote the mass eigenstate, that is, the eigenstate of $M_{N}$, and implicitly $M_{N i j}=M_{i} \delta_{i j}$ is assumed, and the superscript $C$ denotes the charge conjugation. $\hat{H}_{u}$ and $\hat{H}_{d}$ are the supermultiplets of the two Higgs doublet fields $H_{u}$ and $H_{d}$.

Below the lightest RH seesaw mass scale, the singlet supermultiplets $\hat{N}_{i}^{c}$ containing the RH neutrino fields are integrated out, the Majorana mass term for the $\mathrm{LH}$ neutrinos in the flavor basis is obtained,

$$
\begin{gathered}
\mathcal{L}_{m}^{\nu}=-\frac{1}{2} \nu_{L \alpha}\left(m_{\nu}\right)_{\alpha \beta} \nu_{L \beta}+\text { H.c. }, \\
\left(m_{\nu}\right)_{\alpha \beta}=v_{u}^{2}\left(\lambda_{\nu}\right)_{\alpha i} M_{i}^{-1}\left(\lambda_{\nu}\right)_{i \beta},
\end{gathered}
$$

where $M_{i}=\left(M_{1}, M_{2}, M_{3}\right)$ and $v_{u}$ is vacuum expectation value (VEV) of up-type Higgs field $H_{u}, v_{u}=v \sin \beta$ with $v=174 \mathrm{GeV}$. The matrix $\left(m_{\nu}\right)_{\alpha \beta}$ can be diagonalized by a single unitary matrix-Maki-Nakagawa-Sakatamatrix $-U_{\text {MNS }}$ as 


$$
\left(m_{\nu}\right)=U_{\mathrm{MNS}}^{*} \mathrm{D}_{m_{\nu}} U_{\mathrm{MNS}}^{\dagger},
$$

where $\mathrm{D}_{m_{\nu}}=\operatorname{diag}\left(m_{\nu_{1}}, m_{\nu_{2}}, m_{\nu_{3}}\right)$.

The solar, atmospheric, and reactor neutrino experiments have shown at $3 \sigma$ level that [48]

$$
\begin{aligned}
\Delta m_{12}^{2} & =(6.93-7.96) \times 10^{-5}\left(\mathrm{eV}^{2}\right), \\
\Delta m_{23}^{2} & =(2.42-2.66) \times 10^{-3}\left(\mathrm{eV}^{2}\right), \\
\sin ^{2} \theta_{12} & =(0.250-0.354), \\
\sin ^{2} \theta_{23} & =(0.381-0.615), \\
\sin ^{2} \theta_{13} & =(0.0190-0.0240)
\end{aligned}
$$

Note that in this article we will assume that the mass spectrum of light neutrinos is hierarchical $\left(m_{\nu_{1}} \ll m_{\nu_{2}} \ll m_{\nu_{3}}\right)$ and

$$
\hat{U}=\left(\begin{array}{c}
c_{13} c_{12} \\
-c_{23} s_{12}-s_{23} s_{13} c_{12} e^{i \delta} \\
s_{23} s_{12}-c_{23} s_{13} c_{12} e^{i \delta}
\end{array}\right.
$$

where $c_{i j}=\cos \theta_{i j}, s_{i j}=\sin \theta_{i j}$, and $\delta$ is the Dirac $C P$ violating phase, and $\alpha$ and $\beta$ are two Majorana $C P$ violation phases. The input values of the angles and three $C P$-violation phases at GUT scale are set, respectively, so that at low energy the following values are realized, by

$$
\begin{aligned}
s_{23} & =\sqrt{0.441}, \quad s_{13}=\sqrt{0.02166}, \quad s_{12}=\sqrt{0.306}, \\
\alpha & =0, \quad \beta=0, \quad \delta=261^{\circ} .
\end{aligned}
$$

In addition, we parametrize the matrix of neutrino Yukawa couplings à la Casas-Ibarra [35]

$$
\lambda_{\nu}=\frac{1}{v_{u}} U_{\mathrm{MNS}}^{*} \sqrt{\mathrm{D}_{m_{\nu}}} R \sqrt{M}
$$

where

$R=\left(\begin{array}{ccc}\tilde{c}_{13} \tilde{c}_{12} & \tilde{c}_{13} \tilde{s}_{12} & \tilde{s}_{13} \\ -\tilde{c}_{23} \tilde{s}_{12} & \tilde{c}_{23} \tilde{c}_{12}-\tilde{s}_{23} \tilde{s}_{13} \tilde{s}_{12} & \tilde{s}_{23} \tilde{c}_{13} \\ \tilde{s}_{23} \tilde{s}_{12}-\tilde{c}_{23} \tilde{s}_{13} \tilde{c}_{12} & -\tilde{s}_{23} \tilde{c}_{12}-\tilde{c}_{23} \tilde{s}_{13} \tilde{s}_{12} & \tilde{c}_{23} \tilde{c}_{13}\end{array}\right)$.

We adopt that $R$ is a complex orthogonal matrix, $R^{\mathbf{T}} R=1$, so that $\tilde{c}_{i j}=\cos z_{i j}$ and $\tilde{s}_{i j}=\sin z_{i j}$ with $z_{i j}=$ $x_{i j}+\sqrt{-1} y_{i j}$ because we will calculate $C P$-violating process such as Leptogensis. thus $m_{\nu_{3}} \simeq \sqrt{\Delta m_{\mathrm{atm}}^{2}}$ and $m_{\nu_{2}} \simeq \sqrt{\Delta m_{\odot}^{2}}$ and also that all mixing angles lie in the interval $0<\theta_{12}, \theta_{23}$, $\theta_{13}<\pi / 2$. Furthermore, the lightest LH neutrino mass is fixed, for our main result, to be

$$
m_{\nu_{1}}=0.001(\mathrm{eV})
$$

as we will see that we have no solution of the degenerate case.

We will use the standard parametrization of the MNS matrix,

$$
U_{\mathrm{MNS}}=\hat{U} \operatorname{diag}\left(1, e^{i \alpha}, e^{i \beta}\right),
$$

with

$$
\left.\begin{array}{cc}
c_{13} s_{12} & s_{13} e^{-i \delta} \\
c_{23} c_{12}-s_{23} s_{13} s_{12} e^{i \delta} & s_{23} c_{13} \\
-s_{23} c_{12}-c_{23} s_{13} s_{12} e^{i \delta} & c_{23} c_{13}
\end{array}\right),
$$

\section{COSMOLOGICAL CONSTRAINT}

For our analysis we take into account three types of cosmological observables; (i) dark matter abundance (ii) light element abundances (iii) baryon asymmetry of the Universe. We show our strategy to find favored parameter space from a standpoint of these observables in the CMSSM with seesaw mechanism.

\section{A. Number densities of dark matter and of long-lived slepton}

We consider the neutralino-slepton coannihilation scenario in the framework of CMSSM wherein the LSP is the Bino-like neutralino $\tilde{\chi}_{1}^{0}$ and the NLSP is the lightest slepton $\tilde{\ell}_{1}$ that almost consists of RH stau including tiny flavor mixing,

$$
\tilde{\ell}_{1}=\sum_{f=e, \mu, \tau} C_{f} \tilde{f}
$$

where $C_{e}^{2}+C_{\mu}^{2}+C_{\tau}^{2}=1$, and each interaction state is

$$
\tilde{f}=\cos \theta_{f} \tilde{f}_{L}+\sin \theta_{f} \tilde{f}_{R}
$$

The flavor mixing $C_{f}$ and left-right mixing angle $\theta_{f}$ are determined by solving RG equations with neutrino Yukawa. In our scenario $C_{\tau} \sim 1 \gg C_{e}, C_{\mu}$ and $\sin \theta_{\tau} \sim 1$.

The standard calculation for relic density of the $\tilde{\chi}_{1}^{0}$ leads to an overabundant dark matter density. A tight mass degeneracy between $\tilde{\ell}_{1}$ and $\tilde{\chi}_{1}^{0}$ assists in maintaining the 
chemical equilibrium of SUSY particles with the SM sector and can reduce the relic density below the Planck bound [3]. This is called the coannihilation mechanism [20].

In a unique parameter space where the neutralino-slepton coannihilation works well, we focus on the parameter space where the mass difference between $\tilde{\chi}_{1}^{0}$ and $\tilde{\ell}_{1}$ is smaller than the tau mass,

$$
\delta m \equiv m_{\tilde{\ell}_{1}}-m_{\tilde{\chi}_{1}^{0}}<m_{\tau} .
$$

Assuming flavor conservation, i.e., $\tilde{\ell}_{1}$ is purely $\mathrm{RH}$ stau, open decay channels of $\tilde{\ell}_{1}$ are

$\tilde{\ell}_{1} \rightarrow \tilde{\chi}_{1}^{0} \nu_{\tau} \pi, \quad \tilde{\ell}_{1} \rightarrow \tilde{\chi}_{1}^{0} \nu_{\tau} a_{1}, \quad \tilde{\ell}_{1} \rightarrow \tilde{\chi}_{1}^{0} \nu_{\tau} \rho$,

$\tilde{\ell}_{1} \rightarrow \tilde{\chi}_{1}^{0} \nu_{\tau} \ell \bar{\nu}_{\ell} \quad(\ell \ni e, \mu)$,

where $\pi, a_{1}$, and $\rho$ are light mesons. Due to the phase space suppression and higher-order coupling, the $\tilde{\ell}_{1}$ becomes a long-lived particle $[21,23]$. If the lepton flavor is violated, the following two-body decays are allowed,

$$
\tilde{\ell}_{1} \rightarrow \tilde{\chi}_{1}^{0} \ell \quad(\ell \ni e, \mu) .
$$

In fact, the longevity depends on the degeneracy in mass and also on the magnitude of lepton flavor violation $[49,50]$. As we will see in Sec. III B, we have to assume $\delta m<m_{\mu}$, so the main decay mode is the two-body decay $\tilde{\ell}_{1} \rightarrow \tilde{\chi}_{1}^{0} e$ and therefore the lifetime of the slepton $\tau_{\tilde{l}}$ is given by

$$
\tau_{\tilde{l}} \simeq \frac{8 \pi}{g^{2} \tan ^{2} \theta_{W}} \frac{m_{\tilde{l}}}{(\delta m)^{2}} \frac{1}{\cos ^{2} \theta_{e}+4 \sin ^{2} \theta_{e}} \frac{1}{C_{e}^{2}}
$$

up to leading order of $(\delta m)^{2}$, where $g$ is the gauge coupling of $S U(2)$ and $\theta_{W}$ is the Weinberg angle, respectively.

The long-lived $\tilde{\ell}_{1}$ has significant effect on light element abundances through exotic nuclear processes in the BBN era. To quantitatively determine this effect, we evaluate the number density of $\tilde{\ell}_{1}$ on the era. As we will see, it is closely related with the relic density of $\tilde{\chi}_{1}^{0}$ and it depends on not only $\delta m$ but also on the magnitude of lepton flavor violation. Here, we take decoupling limit of SUSY particles except for $\tilde{\chi}_{1}^{0}$ and $\tilde{\ell}_{1}$.

\section{Dark matter relic density}

After SUSY particles $\left(\tilde{\chi}_{1}^{0}\right.$ and $\left.\tilde{\ell}_{1}\right)$ are chemically decoupled from SM sectors, their total density, $n=n_{\tilde{\chi}_{1}^{0}}+n_{\tilde{\ell}_{1}^{-}}+n_{\tilde{\ell}_{1}^{+}}$, will be frozen. Since all of SUSY particles eventually decays into the LSP $\tilde{\chi}_{1}^{0}$, so that the dark matter relic density is indeed the total density. We find the Boltzmann equation of the total density by adding each one of $n_{\tilde{\chi}_{1}^{0}}$ and $n_{\tilde{\ell}_{1}^{ \pm}}[20,51]$,

$$
\frac{d Y_{n}}{d z}=\frac{-s}{H z} \sum_{i, j=\tilde{\chi}_{1}^{0}, \tilde{\ell}_{1}^{ \pm}}\langle\sigma v\rangle_{i j \rightarrow \mathrm{SM}}\left[Y_{i} Y_{j}-Y_{i}^{e q} Y_{j}^{e q}\right]
$$

where $z=m_{\tilde{\chi}_{1}^{0}} / T, Y_{i}=n_{i} / s$ is the number density of a species $i$ normalized to the entropy density $s$, and $Y_{n}=n / s$, respectively. Here $H$ denotes the Hubble expansion rate, $\langle\sigma v\rangle_{i j \rightarrow \mathrm{SM}}$ represents thermally averaged cross section for an annihilation channel $i j \rightarrow \mathrm{SM}$ particles. Relevant processes and the cross sections are given in Ref. [52]. We search for favored parameters by numerically solving the equation to fit $n$ to the observed dark matter density [48]

$$
0.1126 \leq \frac{m_{\tilde{\chi}_{1}^{0}} n h^{2}}{\rho_{c}} \leq 0.1246 \quad(3 \sigma \text { C.L. }),
$$

where $h=0.678$ is the Hubble constant normalized to $H_{0}=100 \mathrm{kms}^{-1} \mathrm{Mpc}^{-1}$, and $\rho_{c}=1.054 \times 10^{-5} \mathrm{GeVcm}^{-3}$ is the critical density of the Universe.

\section{Number density of long-lived slepton}

Even after the chemical decoupling, although the total density remains the current dark matter density, the ratio of each number density of $\tilde{\chi}_{1}^{0}, \tilde{\ell}_{1}^{-}$, and $\tilde{\ell}_{1}^{+}$continues to evolve. As long as the kinetic equilibrium with the SM sector is maintained, $\tilde{\ell}_{1}$ and $\tilde{\chi}_{1}^{0}$ follow the Boltzmann distribution, and hence $\tilde{\ell}_{1}^{-}$number density until the kinetic decoupling is

$$
n_{\tilde{\ell}_{1}^{-}}=\frac{n_{\tilde{\ell}_{1}^{-}}}{n_{\tilde{\chi}_{1}^{0}}} \frac{n_{1}^{0}}{n} n=e^{-\delta m / T} \frac{n}{2\left(1+e^{-\delta m / T}\right)} .
$$

We focus on the parameter space where $\delta m<m_{\mu}, m_{\mu}$ being the muon mass. Then the lifetime of $\tilde{\ell}_{1}$ is long enough, and we are able to solve the ${ }^{7} \mathrm{Li}$ and ${ }^{6} \mathrm{Li}$ problems $[30,53]$. Processes maintaining the kinetic equilibrium in the space are

$$
\begin{array}{rlrl}
\tilde{\ell}_{1}^{ \pm} \gamma & \leftrightarrow \tilde{\chi}_{1}^{0} \tau^{ \pm}, & \tilde{\ell}_{1}^{ \pm} \gamma & \leftrightarrow \tilde{\chi}_{1}^{0} \mu^{ \pm}, \\
\tilde{\ell}_{1}^{ \pm} \tau^{\mp} & \leftrightarrow \tilde{\chi}_{1}^{0} \gamma, \quad \tilde{\ell}_{1}^{ \pm} \mu^{\mp} \leftrightarrow \tilde{\chi}_{1}^{0} \gamma, \quad \tilde{\ell}_{1}^{ \pm} e^{\mp} \leftrightarrow \tilde{\chi}_{1}^{0} \gamma .
\end{array}
$$

Even for a tiny lepton flavor violation (LFV), flavor changing processes are relevant due to much larger densities of $e$ and $\mu$ compared with that of $\tau$ for the Universe temperature smaller than $m_{\tau}$. For example, for the universe temperature $T=70 \mathrm{MeV}$, reaction rates of these processes are

\footnotetext{
${ }^{1}$ Note that the process $\tilde{\ell}_{1}^{ \pm} \gamma \leftrightarrow \tilde{\chi}_{1}^{0} e^{ \pm}$must not be included. The process should be incorporated into a corrective part of the decay (inverse decay) $\tilde{\ell}_{1}^{ \pm} \leftrightarrow \tilde{\chi}_{1}^{0} e^{ \pm}$. Similarly, if the decay $\tilde{\ell}_{1}^{ \pm} \leftrightarrow \tilde{\chi}_{1}^{0} \mu^{ \pm}$ is open, the process $\tilde{\ell}_{1}^{ \pm} \gamma \leftrightarrow \tilde{\chi}_{1}^{0} \mu^{ \pm}$also must not be taken into account.
} 


$$
\begin{aligned}
& \frac{\left\langle\sigma^{\prime} v\right\rangle_{\tilde{e}_{1} e \leftrightarrow \tilde{\chi}_{1}^{0} \gamma} n_{e}}{\left\langle\sigma^{\prime} v\right\rangle_{\tilde{\ell}_{1} \tau \leftrightarrow \tilde{\chi}_{1}^{0} \gamma} n_{\tau}} \simeq\left(1.08 \times 10^{9}\right) C_{e}^{2}, \\
& \frac{\left\langle\sigma^{\prime} v\right\rangle_{\tilde{\ell}_{1} \mu \leftrightarrow \tilde{x}_{1}^{0} \gamma} n_{\mu}}{\left\langle\sigma^{\prime} v\right\rangle_{\tilde{\ell}_{1} \tau \leftrightarrow \tilde{\chi}_{1}^{0} \gamma} n_{\tau}} \simeq\left(9.93 \times 10^{7}\right) C_{\mu}^{2} .
\end{aligned}
$$

Here $\sigma^{\prime}$ represents the cross section of relevant processes for kinetic equilibrium. As long as $C_{e} \gtrsim 3.2 \times 10^{-5}$ and $C_{\mu} \gtrsim 1.0 \times 10^{-4}$, flavor changing processes maintain the kinetic equilibrium, and hence reduce $n_{\tilde{\ell}_{1}^{-}}$. This means that such a small flavor mixing can decrease $n_{\tilde{\ell}_{1}^{-}}$significantly.

The kinetic decoupling is determined by solving coupled Boltzmann equations for $\tilde{\chi}_{1}^{0}, \tilde{\ell}_{1}^{-}$, and $\tilde{\ell}_{1}^{+}$with the initial condition Eq. (20) [54],

$$
\begin{aligned}
\frac{d Y_{\tilde{\chi}}}{d z}= & -\frac{1}{H z} \sum_{i \neq \tilde{\chi}_{1}^{0}}\left\{s\left\langle\sigma^{\prime} v\right\rangle_{\tilde{\chi}_{1}^{0} X \leftrightarrow i Y}\left[Y_{\tilde{\chi}} Y_{X}^{e q}-Y_{i} Y_{Y}^{e q}\right]\right. \\
& \left.+\langle\Gamma\rangle\left[Y_{\tilde{\chi}_{1}^{0}}\left(s Y_{X}^{e q}\right)\left(s Y_{X}^{e q}\right) \ldots-Y_{i}\right]\right\}, \\
\frac{d Y_{\tilde{\ell}_{1}^{ \pm}}}{d z}= & -\frac{1}{H z} \sum_{i \neq \tilde{\ell}_{1}^{ \pm}}\left\{s\left\langle\sigma^{\prime} v\right\rangle_{\tilde{\ell}_{1}^{ \pm} X \leftrightarrow i Y}\left[Y_{\tilde{\ell}_{1}^{ \pm}} Y_{X}^{e q}-Y_{i} Y_{Y}^{e q}\right]\right. \\
& \left.+\langle\Gamma\rangle\left[Y_{\tilde{\ell}_{1}^{ \pm}}-Y_{\tilde{\chi}_{1}^{0}}\left(s Y_{X}^{e q}\right)\left(s Y_{X}^{e q}\right) \ldots\right]\right\} .
\end{aligned}
$$

Here $\Gamma$ represents $\tilde{\ell}_{1}$ decay rate of channels in Eqs. (15) and (16).

\section{B. Big-bang nucleosynthesis}

To solve the lithium problem(s), we need a long-lived particle so that it survives until BBN starts, more precisely synthesis of ${ }^{7} \mathrm{Be}$ begins. Fortunately, our model does have such a long-lived particle, i.e., $\tilde{\ell}_{1}$. This slepton can effectively destruct ${ }^{7} \mathrm{Be}$ which would be ${ }^{7} \mathrm{Li}$ just after the $\mathrm{BBN}$ era. Since at the BBN era would-be ${ }^{7} \mathrm{Li}$ exists as ${ }^{7} \mathrm{Be}$, destructing ${ }^{7} \mathrm{Be}$ effectively means reducing ${ }^{7} \mathrm{Li}$ primordial abundance. This long-lived slepton with degenerate mass can offer the solution to the ${ }^{7} \mathrm{Li}$ problem $[29,30,32,53-61]$. In addition, several articles $[27,62,63]$ report that there are significant amount of ${ }^{6} \mathrm{Li}$ though the standard BBN cannnot predict ${ }^{6} \mathrm{Li}$ abundance.

Since we add the RH Majorana neutrinos, these Yukawa couplings are the seed of LFV, we have another constraint to impose the longevity of the lifetime. To ensure the longevity of the lifetime, only a very tiny electron and muon flavor can mix in the NLSP [23,53]. With keeping these facts in our mind, here we briefly recapitulate how to solve the lithium problem(s).

\section{Nonstandard nuclear reactions in stau-nucleus bound state}

We have constraints for the parameters at low energy so that BBN with the long-lived slepton works well. To see it, we have to take into account the following:

(1) Number density of the slepton at the BBN era

(2) Nonstandard BBN process

(a) Internal conversion $[29,64]$

(b) Spallation [55]

(c) Slepton catalyzed fusion [31]

Number density is calculated by numerically solving Eqs. (24) and (25) if the lifetime is long enough. From this requirement we obtain a constraint $C_{\mu}<\mathcal{O}\left(10^{-5}\right)$ and $C_{e}<\mathcal{O}\left(10^{-7}\right)$ with the assumption $\delta m<m_{\mu}$ [53].

In addition, since its lifetime must be long enough $(\geq 1700 \mathrm{~s})$ there is more stringent constraint on $C_{e}$ with $\delta m$ as has pointed out in Ref. [53].

$$
C_{e} \delta m<3.5 \times 10^{-9} \mathrm{MeV} \text { for } \sin \theta_{e}=0.6 .
$$

\section{Nonstandard nuclear interactions}

Internal conversion. - In a relatively early stage of the $\mathrm{BBN}$, the long-lived slepton forms a bound state with ${ }^{7} \mathrm{Be}$ and ${ }^{7} \mathrm{Li}$ nucleus, respectively. These bound states give rise to internal conversion processes [29],

$$
\begin{aligned}
& \left({ }^{7} \mathrm{Be} \tilde{\ell}_{1}^{-}\right) \rightarrow \tilde{\chi}_{1}^{0}+\nu_{\tau}+{ }^{7} \mathrm{Li}, \\
& \left({ }^{7} \mathrm{Li} \tilde{\ell}_{1}^{-}\right) \rightarrow \tilde{\chi}_{1}^{0}+\nu_{\tau}+{ }^{7} \mathrm{He} .
\end{aligned}
$$

The daughter ${ }^{7} \mathrm{Li}$ nucleus in the process Eq. (27a) is destructed either by an energetic proton or the process (27b) while the daughter ${ }^{7} \mathrm{He}$ nucleus in the process Eq. (27b) immediately decays into ${ }^{6} \mathrm{He}$ nucleus and neutron, then rapid spallation processes by the background particles convert the produced ${ }^{6} \mathrm{He}$ into harmless nuclei, e.g., ${ }^{3} \mathrm{He},{ }^{4} \mathrm{He}$, etc. Hence, the nonstandard chain reactions by the long-lived slepton could yield smaller ${ }^{7} \mathrm{Be}$ and ${ }^{7} \mathrm{Li}$ abundances than those in the standard BBN scenario, which is precisely the requirement for solving the ${ }^{7} \mathrm{Li}$ problem. This is the scenario we proposed.

We find that the time scale of the reaction is much shorter than the $\mathrm{BBN}$ time scale as long as $\delta m$ is larger than several $\mathrm{MeV}$. A parent nucleus is converted into another nucleus immediately once the bound state is formed. The bound state formation makes the interaction between the slepton and a nucleus more efficient by two reasons: First, the overlap of wave functions of the slepton and a nucleus becomes large since these are confined in the small space. Second, the short distance between the slepton and a nucleus allows virtual exchange of the hadronic current even if $\delta m<m_{\pi}$. 
Nonstandard process with bound helium.-The slepton forms a bound state with ${ }^{4} \mathrm{He}$ as well. This fact causes two nonstandard processes. One of these processes is the spallation process of the ${ }^{4} \mathrm{He}$ nucleus [55],

$$
\begin{gathered}
\left({ }^{4} \mathrm{He} \tilde{\ell}_{1}^{-}\right) \rightarrow \tilde{\chi}_{1}^{0}+\nu_{\tau}+\mathrm{t}+\mathrm{n}, \\
\left({ }^{4} \mathrm{He} \tilde{\ell}_{1}^{-}\right) \rightarrow \tilde{\chi}_{1}^{0}+\nu_{\tau}+\mathrm{d}+\mathrm{n}+\mathrm{n}, \\
\left({ }^{4} \mathrm{He} \tilde{\ell}_{1}^{-}\right) \rightarrow \tilde{\chi}_{1}^{0}+\nu_{\tau}+\mathrm{p}+\mathrm{n}+\mathrm{n}+\mathrm{n} .
\end{gathered}
$$

and the other channel is called slepton-catalyzed fusion [31];

$$
\left({ }^{4} \mathrm{He} \tilde{\ell}_{1}^{-}\right)+\mathrm{d} \rightarrow \tilde{\ell}_{1}^{-}+{ }^{6} \mathrm{Li}
$$

Since the LFV coupling and $\delta m$ determines which light elements are overproduced by these nonstandard reactions, we need careful study of the evolution of the slepton- ${ }^{4} \mathrm{He}$ bound state for the parameter space of $C_{\alpha}$ 's and $\delta m$. In general, the spallation process is disastrous. In order to suppress it, $\delta m<30 \mathrm{MeV}$ must be fulfilled.

The catalyzed fusion process enhances the ${ }^{6} \mathrm{Li}$ production [31]. The thermal averaged cross section of the catalyzed fusion is precisely calculated in Refs. [65,66], which is much larger than that of the ${ }^{6} \mathrm{Li}$ production in the standard $\mathrm{BBN},{ }^{4} \mathrm{He}+d \rightarrow{ }^{6} \mathrm{Li}+\gamma$, by 6 to 7 orders of magnitude. The overproduction of the ${ }^{6} \mathrm{Li}$ nucleus by the catalyzed fusion process leads stringent constraints on $(\delta m)^{2} C_{e}^{2}$ from below to make the slepton lifetime shorter than $5000 \mathrm{~s}$ [53]. With the lower bound on the lifetime $1700 \mathrm{~s}$, in addition to the upper bound on $C_{e}$, Eq. (26), we have a lower bound on it. For $\delta m=10 \mathrm{MeV}$ and $\sin \theta_{e}=0.6$,

$1700 \mathrm{~s} \leq \tau_{\tilde{\ell}} \leq 5000 \mathrm{~s} \Leftrightarrow 2.0 \times 10^{-10} \leq C_{e} \leq 3.5 \times 10^{-10}$

is required.

Furthermore, there are several reports [27] that insist there are significant amounts of ${ }^{6} \mathrm{Li}$. If we take these seriously, we can make use of the catalyzed fusion here and, in this case, the slepton lifetime must be between $3500 \mathrm{~s}$ and $5000 \mathrm{~s}$ which corresponds to the requirement

$3500 \mathrm{~s} \leq \tau_{\tilde{e}} \leq 5000 \mathrm{~s} \Leftrightarrow 2.0 \times 10^{-10} \leq C_{e} \leq 2.5 \times 10^{-10}$.

\section{Leptogenesis}

We calculate the lepton asymmetry assuming the RH neutrinos are hierarchical in mass that is generated by the $C P$ asymmetric reactions of the lightest $\mathrm{RH}$ neutrino $N_{1}$ and its superpartner $\tilde{N}_{1}$. Typical parameters for solving the ${ }^{7} \mathrm{Li}$ and ${ }^{6} \mathrm{Li}$ problems are $M_{1} \sim 10^{10} \mathrm{GeV}$ and $\left|\lambda_{\alpha 1}\right| \sim 10^{-3}$. Further, the decay parameter should be $K \equiv \Gamma_{N_{1}} / H\left(M_{1}\right) \sim$ $\mathcal{O}(1)$ and $K_{\alpha} \equiv K \cdot \operatorname{BR}\left(N_{1} \rightarrow \ell_{\alpha} \phi\right) \sim \mathcal{O}(0.1)(\alpha \ni e, \mu, \tau)$. Here, $H\left(M_{1}\right)$ is the Hubble parameter at the temperature $T=M_{1}$. In cases where the leptogenesis in the strong washout regime takes place at $T \lesssim 10^{12} \mathrm{GeV}$ and $K_{\alpha}$ are comparable with each other, the lepton number of each flavor separately evolves, and it gives rise to $\mathcal{O}(1)$ corrections to the final lepton asymmetry with respect to where the flavor effects are ignored $[67,68]$. As studied in Refs. $[69,70]$, the correction could be significant in the SUSY flavored case.

The lepton asymmetry is calculated by a set of the coupled evolution equations of the number densities of $N_{1}, \tilde{N}_{1}$, and the lepton numbers of each flavor. Since the superequilibration is maintained throughout the temperature range we consider [71], the equality of asymmetries of each lepton and its scalar partner is also maintained, and $Y_{B-L}=2 \times\left(Y_{\Delta_{e}}+Y_{\Delta_{\mu}}+Y_{\Delta_{\tau}}\right)$ with $Y_{\Delta_{\alpha}}=B / 3-L_{\alpha}$. In the superequilibration regime, the primary piece of the coupled equations are given as follows [72]

$$
\begin{aligned}
& \frac{d Y_{N_{1}}}{d z}=\frac{-z}{s H\left(M_{1}\right)}\left(\frac{Y_{N_{1}}}{Y_{N_{1}}^{e q}}-1\right)\left[\gamma_{N_{1}}+\gamma_{N_{1}}^{s 1}\right], \\
& \frac{d Y_{\tilde{N}_{+}}}{d z}=\frac{-z}{s H\left(M_{1}\right)}\left(\frac{Y_{\tilde{N}_{+}}}{Y_{\tilde{N}_{1}}^{e q}}-2\right)\left[\gamma_{\tilde{N}_{1}}+\gamma_{\tilde{N}_{1}}^{s 1}\right], \\
& \frac{d Y_{\Delta_{\tilde{N}}}}{d z}=\frac{-z}{s H\left(M_{1}\right)}\left\{\frac{Y_{\Delta_{\tilde{N}}}}{Y_{\tilde{N}_{1}}^{e q}}\left[\gamma_{\tilde{N}_{1}}+\gamma_{\tilde{N}_{1}}^{s 2}\right]\right. \\
& d z=\frac{-z}{s H\left(M_{1}\right)}\left\{\varepsilon_{i}\left(\frac{Y_{N_{1}}}{Y_{N_{1}}^{e q}}-1\right)\left[\gamma_{N_{1}}+\gamma_{N_{1}}^{s 1}\right]\right. \\
&\left.+\frac{Y_{\Delta H_{1}}}{Y_{i}}\left[\frac{Y_{\tilde{N}_{+}}^{e q}}{Y_{H_{1}}^{e q}}-2\right)\left[\gamma_{\tilde{N}_{1}}^{s 4}\right]\right\}, \\
&-\frac{Y_{\Delta \ell}}{Y_{\ell}^{e q}}\left[\left(\frac{1}{2} \gamma_{\tilde{N}_{1}}^{i}+\gamma_{\tilde{N}_{1}}^{s s}\right]\right. \\
&-\frac{Y_{\Delta H_{u}}}{Y_{H_{u}}^{e q}}\left[\left(\frac{1}{2} \gamma_{N_{1}}^{i}+\gamma_{N_{1}}^{s 3}\right)+\left(\gamma_{\tilde{N}_{1}}^{i}+\gamma_{\tilde{N}_{1}}^{s 5}\right)\right]
\end{aligned}
$$

Here, $z=M_{1} / T$. We introduced transformed yield values for $\tilde{N}_{1}, Y_{\tilde{N}_{+}} \equiv Y_{\tilde{N}_{1}}+Y_{\tilde{N}_{1}^{*}}$, and $Y_{\Delta_{\tilde{N}}} \equiv Y_{\tilde{N}_{1}}-Y_{\tilde{N}_{1}^{*}} \cdot \gamma_{N_{1}}$ and $\gamma_{\tilde{N}_{1}}$ are thermally averaged decay rates of $N_{1}$ and $\tilde{N}_{1}$, respectively. $\gamma_{X}^{s n}(n=1,2,3, \ldots)$ symbolizes a combination of thermally averaged cross sections, and the explicit one is shown in the Appendix in Ref. [72]. Relevant cross sections are given in Ref. [73]. Coefficient $C_{\alpha \beta}^{\ell}\left(C_{\beta}^{H}\right)$ is a conversion 
factor from the asymmetry of $\ell_{\alpha}(H)$ to that of $\ell_{\beta}$, $\left(n_{\ell_{\alpha}}-n_{\bar{\ell}_{\alpha}}\right) / n_{\ell_{\alpha}}^{e q}=-\sum_{\beta} C_{\alpha \beta}^{\ell}\left(Y_{\Delta_{\beta}} / Y_{\ell}^{e q}\right)$ and $\left(n_{H}-n_{\bar{H}}\right) /$ $n_{H}^{e q}=-\sum_{\beta} C_{\beta}^{H}\left(Y_{\Delta_{\beta}} / Y_{\ell}^{e q}\right)$. The entries are determined by constraints among the chemical potentials enforced by the equilibrium reactions at the stage where the asymmetries are generated, $T \sim M_{1}$. In our scenario, $M_{1} \sim 10^{10} \mathrm{GeV}$, and $C_{\alpha \beta}^{l}$ and $C_{\beta}^{H}$ are [72]

$$
\begin{aligned}
C_{\alpha \beta}^{l} & =\frac{1}{3 \times 2148}\left(\begin{array}{ccc}
906 & -120 & -120 \\
-75 & 688 & -28 \\
-75 & -28 & 688
\end{array}\right), \\
C^{H} & =\frac{1}{2148}\left(\begin{array}{lll}
37 & 52 & 52
\end{array}\right) .
\end{aligned}
$$

The $C P$ asymmetry receives contributions from not only the RH neutrinos but also its scalar partner. The flavor dependent $C P$ asymmetry for the channel $N_{i} \rightarrow \ell_{\alpha} \phi$ is defined as

$$
\varepsilon_{\alpha}^{i} \equiv \frac{\Gamma\left(N_{i} \rightarrow \ell_{\alpha} \phi\right)-\Gamma\left(N_{i} \rightarrow \bar{\ell}_{\alpha} \phi^{\dagger}\right)}{\Gamma\left(N_{i} \rightarrow \ell_{\alpha} \phi\right)+\Gamma\left(N_{i} \rightarrow \bar{\ell}_{\alpha} \phi^{\dagger}\right)}
$$

and is obtained as [74]

$$
\begin{aligned}
& \varepsilon_{\alpha}^{i}=\varepsilon_{\alpha}^{i}(\text { vertex })+\varepsilon_{\alpha}^{i}(\text { wave }), \\
& \varepsilon_{\alpha}^{i}(\text { vertex })=-\frac{1}{8 \pi} \sum_{j} \frac{M_{j}}{M_{i}} \log \left[1+\frac{M_{i}^{2}}{M_{j}^{2}}\right] \frac{\mathfrak{I}\left[\left(\lambda^{\dagger} \lambda\right)_{j i} \lambda_{\beta i}^{*} \lambda_{\alpha i}\right]}{\left(\lambda^{\dagger} \lambda\right)_{i i}}, \\
& \varepsilon_{\alpha}^{i}(\text { wave })=-\frac{2}{8 \pi} \sum_{j} \frac{M_{i}}{M_{j}^{2}-M_{i}^{2}} \\
& \times \frac{\Im\left\{\left[M_{j}\left(\lambda^{\dagger} \lambda\right)_{j i}+M_{i}\left(\lambda^{\dagger} \lambda\right)_{i j}\right] \lambda_{\beta i}^{*} \lambda_{\alpha i}\right\}}{\left(\lambda^{\dagger} \lambda\right)_{i i}} .
\end{aligned}
$$

The $C P$ asymmetries for other channels, $N_{i} \rightarrow \tilde{l}_{\alpha} \tilde{\chi}$, $\tilde{N}_{i} \rightarrow l_{\alpha} \tilde{\chi}$, and $\tilde{N}_{i} \rightarrow \tilde{l}_{\alpha} \phi$, are defined similarly and given as the same results with Eqs. (38), (39), and (40).

The lepton and slepton asymmetry converts to the baryon asymmetry, and the conversion factor in MSSM scenarios is $Y_{B}=(8 / 23) Y_{B-L}$ [75]. The required lepton asymmetry in 3 sigma range is

$$
2.414 \times 10^{-10} \lesssim\left|Y_{B-L}\right| \lesssim 2.561 \times 10^{-10}
$$

for the observed baryon number $\Omega_{b} h^{2}=0.0223 \pm 0.0002$ $(1 \sigma)$ [48].

Figure 1 shows the evolution of lepton number for a typical parameter obtained in this study. Numerical computations in this work are performed by using the complete set of coupled Boltzmann equations. For illustrating

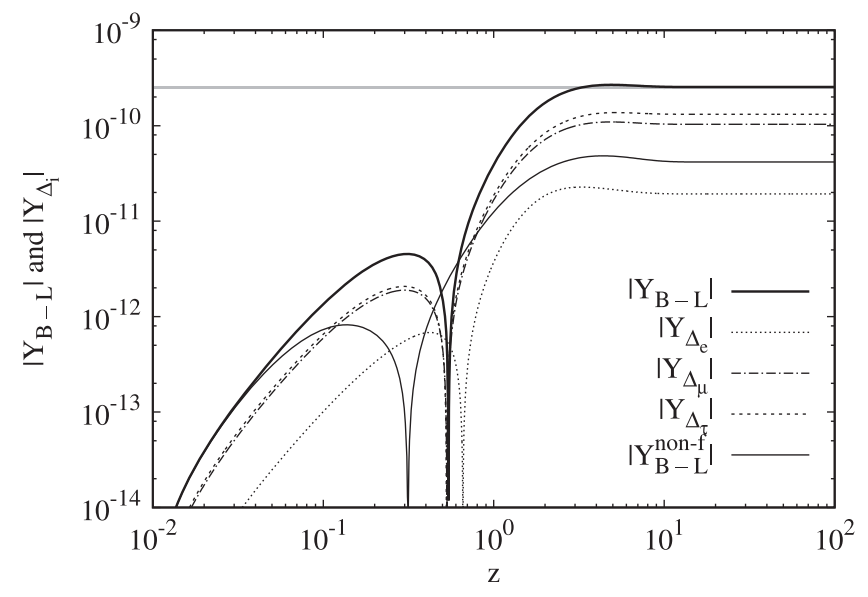

FIG. 1. Evolutions of $\left|Y_{B-L}\right|$ and each lepton asymmetry $\left|Y_{\Delta_{i}}\right|$ for a typical parameter in this paper. Horizontal band (gray) corresponds to the observed baryon asymmetry. $\left|Y_{B-L}^{\text {non-f }}\right|$ shows the lepton asymmetry in the absence of flavor effect.

the importance of flavor effect, we also plot the nonflavored result with thin solid line. We find $\mathcal{O}(1)$ correction to the final lepton asymmetry depending on the presence of the flavor effect. Since this correction is introduced into the expected relation between $M_{1}$ and $\lambda_{\alpha i}$, the flavor effects are critical ingredients to understand the correlation among the BBN, the BAU, and the charged LFV in our scenario.

\section{ANALYSIS}

\section{A. Parameter space}

Soft SUSY breaking term in the Lagrangian $\mathcal{L}_{\text {soft }}$ contains more than one hundred parameters in general. In order to perform a phenomenological study, we make an assumption that three gauge couplings are unified at the GUT scale. At that scale, we presume that there exists a universal gaugino mass, $m_{1 / 2}$. Besides, the scalar soft breaking part of the Lagrangian depends only on a common scalar mass $m_{0}$ and trilinear coupling $A_{0}$, in addition on the ratio of VEVs, $\tan \beta$. After fixing a sign ambiguity in the Higgsino mixing parameter $\mu$, we complete five SUSY parameter space of the CMSSM:

$$
m_{1 / 2}, \quad m_{0}, \quad A_{0}, \quad \tan \beta, \quad \operatorname{sign}(\mu) .
$$

Note that we have demonstrated our numerical analyses only in the $\operatorname{sign}(\mu)>0$ case.

In the neutrino Yukawa couplings, Eq. (10), there are 18 parameters since the matrix is $3 \times 3$ complex matrix. We use the low-energy observed quantifies (i) three LH neutrino masses $m_{\nu_{1}}, m_{\nu_{2}}, m_{\nu_{3}}$, (ii) three mixing angles $\sin \theta_{23}, \sin \theta_{13}, \sin \theta_{12}$ in $U_{\text {MNS }}$ [Eq. (8)], and (iii) three $C P$ violating phases $\alpha, \beta, \delta$ [Eq. (9)] as input parameters. They are given in Sec. II. There are nine model parameters, which we express in terms of three RH Majorana neutrino 
masses $M_{1}, M_{2}, M_{3}$ at GUT scale and the remaining three complex angles in the $R$ matrix. Thus, there are a total of nine free parameters and nine experimentally "observed" data in the Dirac-Yukawa couplings.

The low-energy SUSY spectra and the low-energy flavor observables were computed by means of the SPheno-3.3.8 [76,77] using two-loop beta functions with an option of the precision as quadrupole because the slepton flavor mixing is required to be $10^{-12}$ order or even smaller. During these computations, we apply the set of constraints displayed in Table I. We generate SLHA format files and send them to micrOMEGAs_4.3.5 [78-80] which computes the neutralino relic density $\Omega h^{2}$ and the spin-independent scattering cross section with nucleons, as we will briefly mention below.

\section{B. Determining input parameters}

In this subsection, we discuss in detail how we have investigated a very wide range of parameter space. In principle, we must set all the parameters simultaneously so that all the requirements are fulfilled. However, conceptually, we can set the parameters step by step with the small correction from the following steps.

\section{The CMSSM parameters}

Let us start with the constraints on the lightest neutralino mass from relic abundance. For our analysis, we take into account cosmological data - dark matter abundance-that arise from the Planck satellite analysis [2]. In this article, the neutralino relic density, $\Omega h^{2}$, must satisfy the 3 sigma range: $\Omega h^{2} \in[0.1126,0.1246]$ [48]. In CMSSM type theory, the lightest neutralino mass will be of order of $400 \mathrm{GeV}$. In the framework of MSSMRN which we consider, the lightest neutralino mass becomes about $380 \mathrm{GeV}$. What is more, we fix the mass difference $\delta m=0.01 \mathrm{GeV}$ as already studied [53]; furthermore, we decide to use $\tan \beta=25$ because with this value we can easily obtain the right amount of the relic density which must be within the 3 sigma rage of cosmological data. Accordingly, the three SUSY parameters, $m_{1 / 2}, A_{0}$, and $\tan \beta$, are set at the following values:

TABLE I. The experimental constraints.

\begin{tabular}{lcc}
\hline \hline Quantity & & Reference \\
\hline$\Omega h^{2}$ & {$[0.1126,0.1246]$} & {$[48]$} \\
$m_{h}$ & $(124.4,125.8) \mathrm{GeV}$ & {$[48]$} \\
$\mathrm{BR}(B \rightarrow s \gamma)$ & {$[2.82,3.29] \times 10^{-4}$} & {$[81]$} \\
$\mathrm{BR}\left(B_{s} \rightarrow \mu^{+} \mu^{-}\right)$ & $2.8_{-1.8}^{+2.1} \times 10^{-9}$ & {$[82]$} \\
$\operatorname{BR}\left(B_{u} \rightarrow \tau \bar{\nu}\right)$ & $0.52<R_{B \tau \nu}<2.61$ & {$[83]$} \\
$a_{\mu}$ & {$[1.97,50.2] \times 10^{-10}$} & {$[84]$} \\
\hline \hline
\end{tabular}

$$
\begin{aligned}
& m_{1 / 2}=887.0(\mathrm{GeV}), \quad A_{0}=-3090(\mathrm{GeV}), \\
& \tan \beta=25 .
\end{aligned}
$$

At this moment, four SUSY parameters have been fixed including the sign of the $\mu$ term. The remaining parameter is the universal scalar mass, $m_{0}$, which must lie at

$$
m_{0} \approx[707.3,707.4](\mathrm{GeV}),
$$

depending on the mass hierarchy structure of the RH Majorana neutrino sector for fixing the value of $\delta m=0.01 \mathrm{GeV}$.

Note that we have taken into account the logalismical corrections of the corresponding scales and also the slepton mass running effect which are caused by the Dirac-Yukawa beta function. However, these effects are negligible for the dark matter relic density calculations.

It is important to mention that with the above given values of SUSY parameters we obtain the SM-like Higgs mass about $125 \mathrm{GeV}$, i.e., the "right" combination of the values of $\tan \beta, A_{0}$ and stop mass generated by the universal scalar mass $m_{0}$ are selected in our calculation processes.

We show here an example parameter in the top panel of Table II. With this parameter set, the flavor mixing $C_{e}, C_{\mu}$, and the mixing angle $\sin \theta_{e}$, and the lifetime of slepton are calculated. The results are listed in the middle-panel of Table II. It is clear that our model with these parameters solve also both ${ }^{6} \mathrm{Li}$ and ${ }^{7} \mathrm{Li}$ problems. Furthermore, we have calculated the observed quantities, the relic density of dark matter, mass of dark matter, and the mass difference between the NLSP and LSP, $\delta m$, with same parameters. The results are displayed in the bottom-panel of Table II. As has been noted, our result, $\Omega h^{2}=0.1154$, satisfies the relic density obtained from the Planck satellite analysis [2].

\section{The Yukawa coupling}

In order to find a set of parameters with which our model-MSSMRH with boundary condition at the GUT scale-we have performed parameter scan in the following "systematic" way. Essentially we do not scan all mass range of the RH Majorana neutrinos, but we fix the mass ratio of these particles. It means that the second heaviest and the heaviest RH Majorana masses are given by a function of the lightest RH one, hereby we fix the ratio $M_{3} / M_{1}=40$, and we investigate the following three scenarios in this article. Namely,

(1) $M_{2}=2 \times M_{1}, M_{3}=40 \times M_{1}$

(2) $M_{2}=4 \times M_{1}, M_{3}=40 \times M_{1}$

(3) $M_{2}=10 \times M_{1}, M_{3}=40 \times M_{1}$;

i.e., only the ratios of $M_{2} / M_{1}$ are different in each setup.

Fixing the mass of the lightest RH Majorana neutrino and arranging the elements of the complex orthogonal matrix $R$, i.e., the real part of the complex angles $\left(x_{12}, x_{13}, x_{23}\right)$ and the complex part of $y_{12}, y_{13}, y_{23}$, we are now able to 
TABLE II. The example of input parameters and output parameters.

\begin{tabular}{|c|c|}
\hline Input parameters & Value \\
\hline$m_{0}$ & $707(\mathrm{GeV})$ \\
\hline$m_{1 / 2}$ & $887(\mathrm{GeV})$ \\
\hline$A_{0}$ & $-3089(\mathrm{GeV})$ \\
\hline $\tan \beta$ & 25 \\
\hline$\mu /|\mu|$ & +1 \\
\hline$m_{\nu_{1 L}}$ & $10^{-3}(\mathrm{eV})$ \\
\hline$m_{\nu_{2 L}}$ & $4.04 \times 10^{-3}(\mathrm{eV})$ \\
\hline$m_{\nu_{3 L}}$ & $1.18 \times 10^{-2}(\mathrm{eV})$ \\
\hline$M_{1}$ & $2.0 \times 10^{10}(\mathrm{GeV})$ \\
\hline$M_{2}$ & $8.0 \times 10^{10}(\mathrm{GeV})$ \\
\hline$M_{3}$ & $8.0 \times 10^{11}(\mathrm{GeV})$ \\
\hline$\alpha$ & 0 \\
\hline$\beta$ & 0 \\
\hline$\delta$ & 261 \\
\hline$x_{12}$ & 2.28948 \\
\hline$x_{13}$ & 3.56000 \\
\hline$x_{23}$ & 4.80532 \\
\hline$y_{12}$ & 1.02 \\
\hline$y_{13}$ & 0.1 \\
\hline$y_{23}$ & 0.1 \\
\hline Output parameters & Value \\
\hline$C_{e}$ & $3.28 \times 10^{-10}$ \\
\hline & $2.94 \times 10^{-6}$ \\
\hline $\sin \theta_{e}$ & 0.188 \\
\hline$\tau_{\tilde{l}}$ & $4217(\mathrm{~s})$ \\
\hline Output parameters & Value \\
\hline$\Omega h^{2}$ & 0.115 \\
\hline$m_{\tilde{\chi}_{1}^{0}}$ & $379.6(\mathrm{GeV})$ \\
\hline$\delta m$ & $1.01 \times 10^{-2}(\mathrm{GeV})$ \\
\hline
\end{tabular}

calculate the baryon asymmetry. For simplicity, we fix the values of $y_{23}=y_{13}=0.1$ and vary only $y_{12}$ in the complex part of the mixing angles. The real part of complex angles, $x_{12}, x_{13}, x_{23}$, are obtained through the electron mixing in slepton mass matrix, $C_{e}$. To have a enough lifetime of slepton for solving the lithium problem, only extremely narrow ranges of $x_{12}, x_{13}, x_{23}$ - of the order of $10^{-5}$-are allowed because of $C_{e}$ being of the order of $10^{-10}$. To illustrate how the real parts of the flavor mixing are determined, we show the lifetime of the slepton in terms of $x_{23}$ in Fig. 2. The RH Majorana mass is taken to $M_{1}=2.0 \times 10^{10} \mathrm{GeV}$ in case 2 . The blue and green bands represent the slepton lifetime required to solve only the ${ }^{7} \mathrm{Li}$ problem, Eq. (30), and both ${ }^{7} \mathrm{Li}$ and ${ }^{6} \mathrm{Li}$ problems, Eq. (31), respectively. The lifetime changes 2 orders of magnitude for the narrow range of $x_{23}$ of order $10^{-5}$. This is because $C_{e} \sim$ $10^{-10}$ is realized due to the fine-tuned cancellation among the LFV terms in renormalization group equation running.

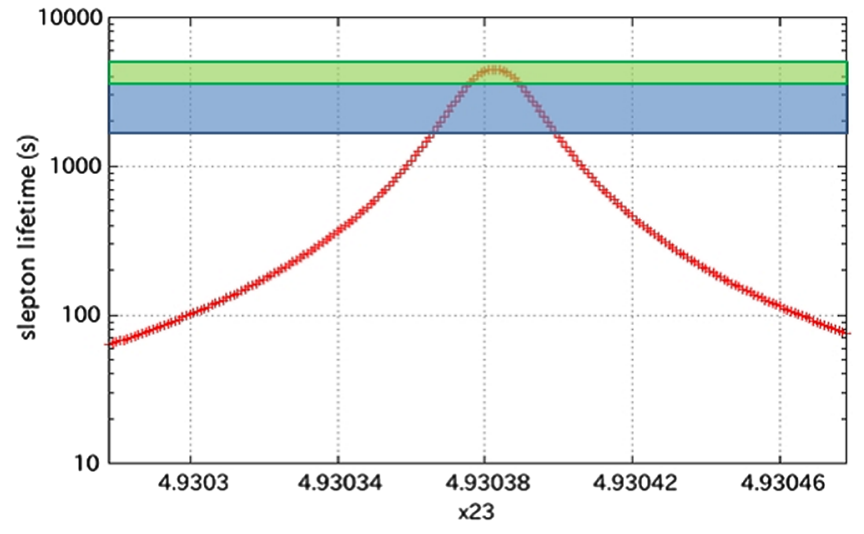

FIG. 2. The lightest slepton lifetime as a function of $x_{23}$. The blue and green band corresponds to the lifetime required to solve the ${ }^{7} \mathrm{Li}$ problem only and both the ${ }^{7} \mathrm{Li}$ and ${ }^{6} \mathrm{Li}$ problems, respectively.

When $x_{23}$ differs from this range, $C_{e}$ is $\mathcal{O}\left(10^{-5}\right)$ and hence the slepton lifetime becomes much shorter. One can see that the real part $x_{23}$ is determined almost uniquely to solve the $\mathrm{Li}$ problems. No need to say that allowed regions of the real part of the complex angles are also depend on the mass structure of the RH Majorana neutrinos thus we have to seek an other tiny parameter space when we change the value of $M_{1}$. Furthermore we check the parameters obtained in this way whether they reproduce the right amount of baryon asymmetry as explained in Sec. III C.

\section{The allowed mass region of the lightest right-handed Majorana neutrino}

We describe our main results in this subsection. First, we discuss the allowed mass range of the lightest RH Majorana neutrino. We have found the upper and lower limit for mass of the lightest RH Majorana neutrino corresponding to its hierarchical structure. With respect to the lithium problem, three cases which we have investigated are listed here:

(1) case of $M_{2}=2 \times M_{1}, M_{3}=40 \times M_{1}$

(a) Taking into account the ${ }^{6} \mathrm{Li}$ and ${ }^{7} \mathrm{Li}$ problem

$$
7.8 \times 10^{8} \leq M_{1} \leq 7.0 \times 10^{10}(\mathrm{GeV})
$$

(b) Taking into account only the ${ }^{7} \mathrm{Li}$ problem

$$
7.8 \times 10^{8} \leq M_{1} \leq 1.0 \times 10^{11}(\mathrm{GeV})
$$

(2) case of $M_{2}=4 \times M_{1}, M_{3}=40 \times M_{1}$

(a) Taking into account the ${ }^{6} \mathrm{Li}$ and ${ }^{7} \mathrm{Li}$ problem

$$
1.9 \times 10^{9} \leq M_{1} \leq 7.0 \times 10^{10}(\mathrm{GeV}) .
$$

(b) Taking into account only the ${ }^{7} \mathrm{Li}$ problem

$$
1.9 \times 10^{9} \leq M_{1} \leq 1.0 \times 10^{11}(\mathrm{GeV}) .
$$



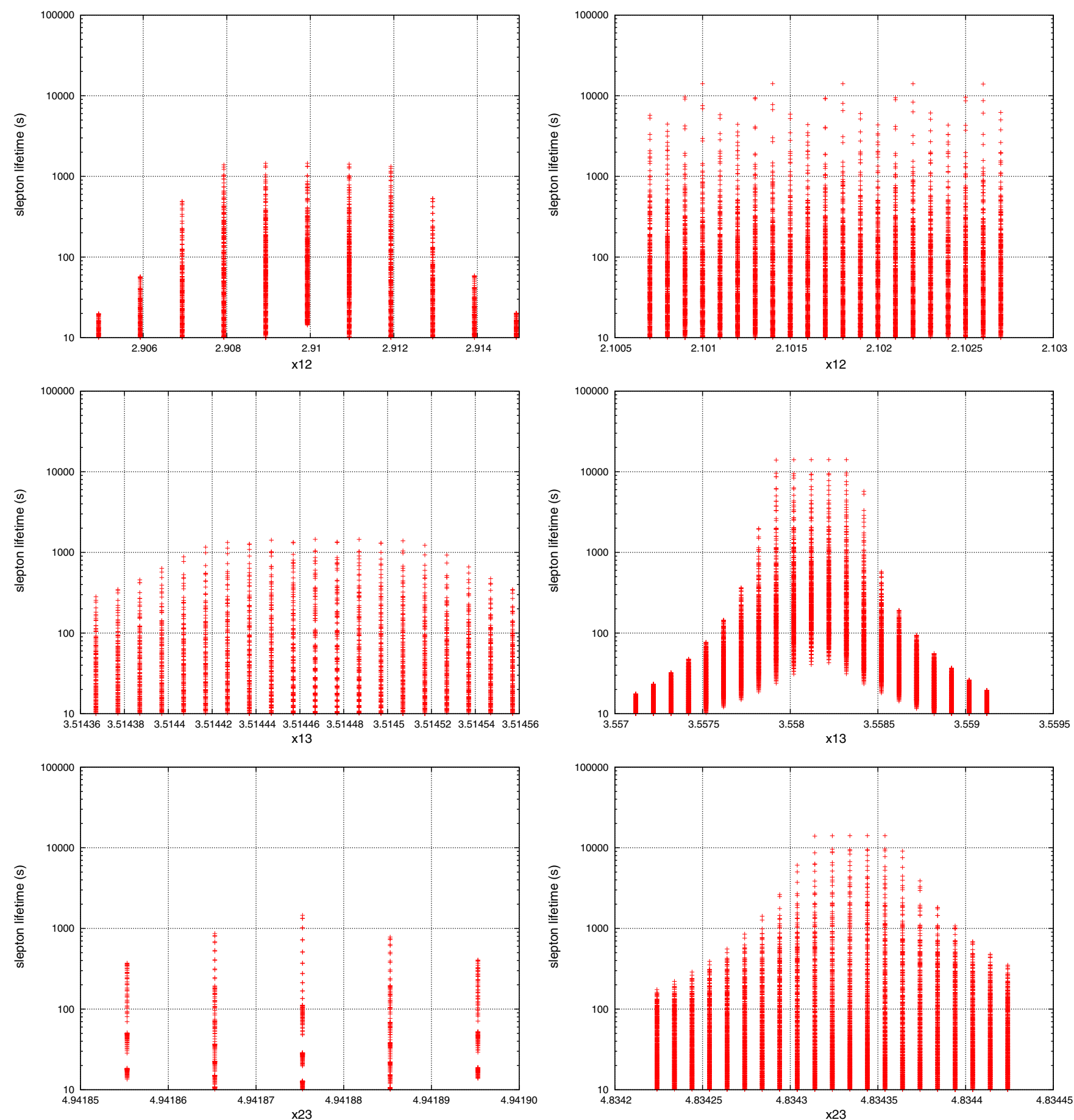

FIG. 3. The slepton lifetime in terms of $x_{12}, x_{13}, x_{23}$ in case 2 . In the left and right panel, $M_{1}$ is taken to $1.2 \times 10^{11} \mathrm{GeV}$ and $3.0 \times 10^{10} \mathrm{GeV}$, respectively.

(3) case of $M_{2}=10 \times M_{1}, M_{3}=40 \times M_{1}$

(a) Taking into account the ${ }^{6} \mathrm{Li}$ and ${ }^{7} \mathrm{Li}$ problem

$$
2.35 \times 10^{9}(\mathrm{GeV}) \leq M_{1} .
$$

One might wonder why we do not write the upper limit of the lightest RH Majorana neutrino mass in the third case. Essentially, we do not need to get the values that definitely exist because the region where the upper limit would be is already excluded by the current experiment date of $\mathrm{BR}(\mu \rightarrow e \gamma)$.

The upper limits of the lightest RH Majoarana neutrino in three different cases are obtained from the limits of $C_{e}$ and $C_{\mu}$, in fact we need to suppress both the slepton mixing $C_{e}$ and $C_{\mu}$. Naively, these flavor mixing are scaled to the Yukawa couplings, so that it is easy to understand why we 
let the absolute values of the Dirac-Yukawa couplings be $\left|\lambda_{\alpha i}\right| \ll 1$ to satisfy the experimental constraints of $C_{e}$ and $C_{\mu}$. At the same time, of course we must satisfy the lowenergy neutrino experiment data, namely $\Delta m^{2}$, and three mixing angles according to Eq. (3) in which we do not consider an extreme fine-tuning in matrix multiplications. Therefore, the eigenvalues of $M_{R}$, or the lightest $\mathrm{RH}$ Majorana neutrino mass since we fix the mass ratios $M_{2} / M_{1}$ and $M_{3} / M_{1}$, should be lighter than the case of $\left|\lambda_{\alpha i}\right| \sim 1$. Further Yukawa coupling constants square is scaled to the RH Majorana neutrino masses, at a certain mass it becomes impossible for $C$ 's to be small enough. Thus, we have the upper bound for $M_{1}$. In Fig. 3, we show the slepton lifetime as a function of $x_{12}, x_{13}, x_{23}$ to illustrate this explanation. The RH Majorana mass $M_{1}$ is taken to $1.2 \times 10^{11} \mathrm{GeV}$ and $3.0 \times 10^{10} \mathrm{GeV}$ in the left and right panels, respectively. In the left panels, the lifetime cannot reach $1700 \mathrm{~s}$ even when $x_{12,13,23}$ are fine-tuned. On the other hand, in the right panels where $M_{1}$ is taken to be smaller, the lifetime can be longer than $1700 \mathrm{~s}$. Note that all of the real parts are determined in very narrow range as we explained in Fig. 2. The flavor mixing $C_{e}$ is more tightly constrained to solve the ${ }^{6} \mathrm{Li}$ problem (or evade ${ }^{6} \mathrm{Li}$ overproduction) and hence the upper bound is more stringent. It is worth noting that with similar reason, we cannot have a degenerate solution for left-handed neutrino mass since in this case also rather large Yukawa coupling is necessary.

On the other hand, to reproduce the matter-antimatter asymmetry generated by leptogenesis so that the $C P$ violating of Majorana decay processes $\varepsilon_{\alpha}^{i}$ [Eq. (37)] should be large than
$10^{-6}$, if one does not take into account the flavor effects neither does not consider also an accidental fine-tuning cancellation in $\lambda_{\nu}^{\dagger} \lambda_{\nu}$. Thus we need a sufficiently large Yukawa couplings and large value of $M_{1}$ is required (as we know in the nonflavor leptogenesis case, $M_{1} \gtrsim 10^{9} \mathrm{GeV}$ [85].) As it is scaled to the RH Majorana neutrino mass at a certain point such a sufficiently large coupling can not be realized.

In concluding, we note that there exists only a tiny allowed parameter space for the lightest RH Majorana neutrino where all experimental data and constraints are fulfilled within the 3 sigma range.

\section{PREDICTIONS FROM PARAMETER SEARCH}

\section{A. Predictions mainly from CMSSM parameters}

As explained in Sec. IV B 1, CMSSM parameter is almost determined uniquely from $m_{\tilde{\chi}_{1}^{0}}, \delta m$, and SM Higgs mass. Therefore the dark matter relic abundance, SUSY mass spectrum, and the contribution to the muon magnetic anomalous moment $g-2$ are more or less predicted uniquely.

In our analysis, the relic abundance of the neutralino density is

$$
\Omega h^{2}=0.115 \text {. }
$$

For calculation of the spin-independent cross section with the nucleon, we use the following values of the quark form-factors in the nucleon which are the default values in the MICROMEGAs code,

TABLE III. SUSY particle masses.

\begin{tabular}{|c|c|c|}
\hline Particle & Mass $(\mathrm{GeV})$ & Mixing \\
\hline $\begin{array}{l}\tilde{d}_{1} \\
\tilde{d}_{2} \\
\tilde{d}_{3} \\
\tilde{d}_{4} \\
\tilde{d}_{5} \\
\tilde{d}_{6}\end{array}$ & $\begin{array}{l}1.453 \times 10^{3} \\
1.696 \times 10^{3} \\
1.850 \times 10^{3} \\
1.851 \times 10^{3} \\
1.925 \times 10^{3} \\
1.926 \times 10^{3}\end{array}$ & $\begin{array}{l}\tilde{d}_{1} \simeq(0.9910-0.0000 i) \tilde{b_{L}}+(0.1289-0.0000 i) \tilde{b}_{R} \\
\tilde{d}_{2} \simeq(0.9916-0.0000 i) \tilde{b_{R}}+(-0.1286+0.0000 i) \tilde{b_{L}} \\
\tilde{d}_{3} \simeq(0.9997+0.0189 i) \tilde{s_{R}}+(0.0068+0.0001 i) \tilde{s_{L}} \\
\tilde{d_{4}} \simeq(-0.9263-0.3766 i) \tilde{d}_{R}+(-0.0003-0.0001 i) \tilde{d}_{L} \\
\tilde{d}_{5} \simeq(-0.9835-0.016 i) \tilde{s_{L}}+(0.1664-0.0588 i) \tilde{d}_{L} \\
\tilde{d}_{6} \simeq(0.8698-0.4605 i) \tilde{d}_{L}+(0.1752-0.0229 i) \tilde{s_{L}}\end{array}$ \\
\hline $\begin{array}{l}\tilde{u}_{1} \\
\tilde{u}_{2} \\
\tilde{u}_{3} \\
\tilde{u}_{4} \\
\tilde{u}_{5} \\
\tilde{u}_{6}\end{array}$ & $\begin{array}{l}8.775 \times 10^{2} \\
1.502 \times 10^{3} \\
1.858 \times 10^{3} \\
1.858 \times 10^{3} \\
1.924 \times 10^{3} \\
1.924 \times 10^{3}\end{array}$ & $\begin{array}{l}\tilde{u_{1}} \simeq(0.9604-0.0000 i) \tilde{t_{R}}+(0.2749-0.0000 i) \tilde{L_{L}} \\
\tilde{u_{2}} \simeq(-0.9603+0.0000 i) \tilde{t_{L}}+(0.2784-0.0000 i) \tilde{t_{R}} \\
\tilde{u_{3}} \simeq(0.9999-0.0001 i) \tilde{c_{R}}+(0.0103+0.0000 i) \tilde{c_{L}} \\
\tilde{u_{4}} \simeq(0.2862+0.9581 i) \tilde{u_{R}}+(0.0000+0.0000 i) \tilde{u_{L}} \\
\tilde{u_{5}} \simeq(0.9958+0.0045 i) \tilde{c_{L}}+(0.0659+0.0618 i) \tilde{u_{L}} \\
\tilde{u_{6}} \simeq(-0.7492+0.6560 i) \tilde{u_{L}}+(0.0092-0.0899 i) \tilde{c_{L}}\end{array}$ \\
\hline $\begin{array}{l}\tilde{l}_{1} \\
\tilde{l}_{2} \\
\tilde{l}_{3} \\
\tilde{l}_{4} \\
\tilde{l}_{5} \\
\tilde{l}_{6}\end{array}$ & $\begin{array}{l}3.796 \times 10^{2} \\
7.806 \times 10^{2} \\
7.817 \times 10^{2} \\
7.980 \times 10^{2} \\
9.215 \times 10^{2} \\
9.219 \times 10^{2}\end{array}$ & $\begin{array}{l}\tilde{l_{1}} \simeq(-0.9852+0.0000 i) \tilde{\tau_{R}}+(-0.1710-0.0000 i) \tilde{\tau_{L}} \\
\tilde{l_{2}} \simeq(-0.6766-0.7360 i) \tilde{\mu_{R}}+(-0.0141-0.0154 i) \tilde{\mu_{L}} \\
\tilde{l_{3}} \simeq(-0.6639+0.7477 i) \tilde{e_{R}}+(0.0000+0.7605 i) \tilde{e_{L}} \\
\tilde{l_{4}} \simeq(0.9852+0.0000 i) \tilde{\tau_{L}}+(-0.1710-0.0000 i) \tilde{\tau_{R}} \\
\tilde{l_{5}} \simeq(0.6681+0.7311 i) \tilde{\mu_{L}}+(0.1077-0.0835 i) \tilde{e_{L}} \\
\tilde{l_{6}} \simeq(-0.7833+0.6064 i) \tilde{e_{L}}+(0.0919+0.1006 i) \tilde{\mu_{L}}\end{array}$ \\
\hline$\tilde{g}$ & $1.986 \times 10^{3}$ & \\
\hline
\end{tabular}



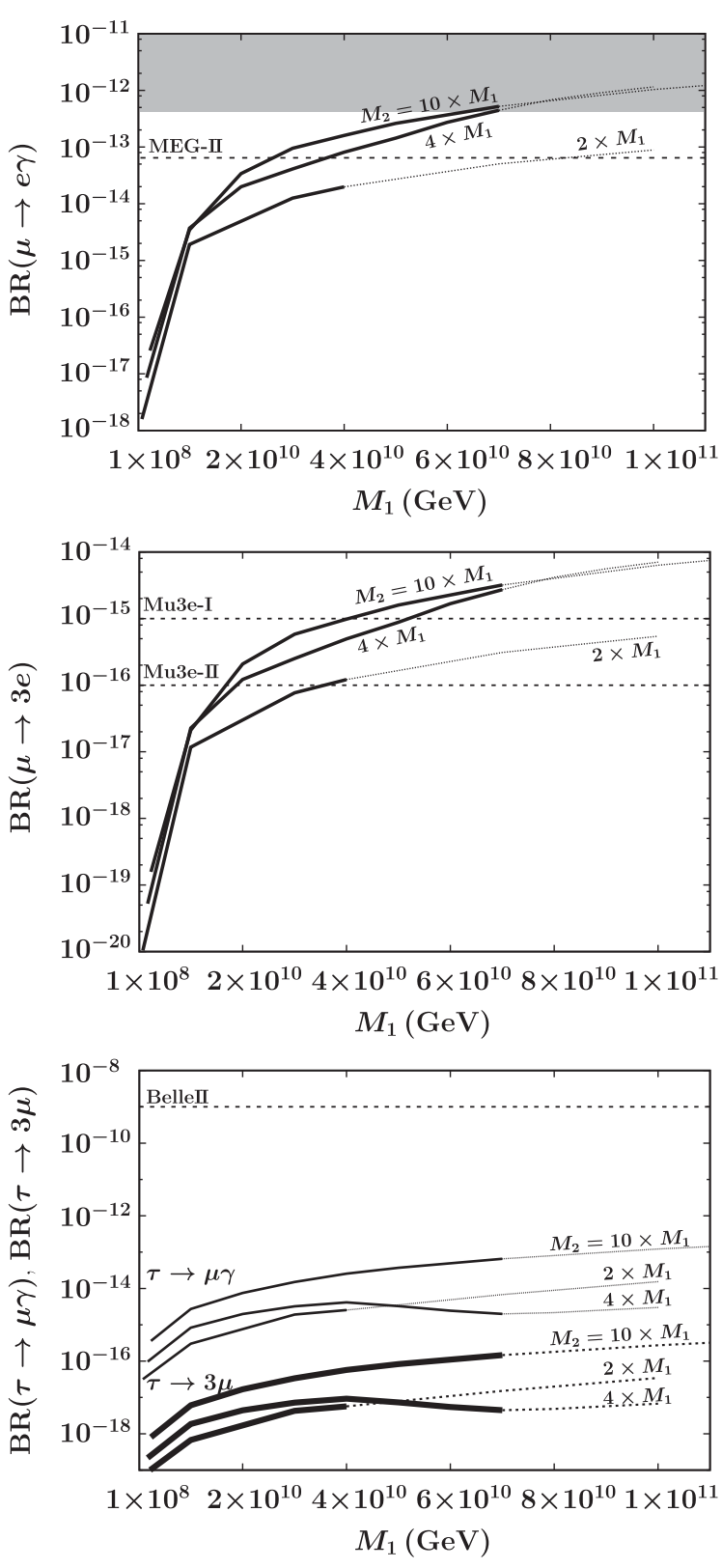

FIG. 4. $\quad \mathrm{BR}(\mu \rightarrow e \gamma), \quad \mathrm{BR}(\mu \rightarrow 3 e), \quad \mathrm{BR}(\tau \rightarrow \mu \gamma), \quad$ and $\operatorname{BR}(\tau \rightarrow 3 \mu)$ as a function of $M_{1}$ for $M_{2}=2 \times M_{1}, 4 \times M_{1}$, and $10 \times M_{1}$. The ${ }^{7} \mathrm{Li}$ problem is solved with parameters in each line, while both the ${ }^{7} \mathrm{Li}$ and ${ }^{6} \mathrm{Li}$ problems are solved only for thick part. Gray region is excluded by MEG experiment, and the horizontal lines show future sensitivity.

$$
\begin{array}{rlrl}
f_{d}^{p} & =0.033, & f_{u}^{p}=0.023, & f_{s}^{p}=0.26, \\
f_{d}^{n}=0.042, & f_{u}^{n}=0.018, & f_{s}^{n}=0.26,
\end{array}
$$

and we get

$$
\sigma^{\mathrm{SI}}=1.05 \times 10^{-47} \mathrm{~cm}^{2},
$$

so that our dark matter candidate satisfies easily the limit of the spin-independent cross section with the nucleon reported by the LUX Collaboration [86], even including the main uncertainty from the strange quark coefficient. If we use another set of quark coefficients (the large corrections to $f_{s}^{p / n}$ ) can lead to a shift by a factor of 2-6 in the spinindependent cross section [78].

Masses of supersymmetric particles are shown in Table III. Note that these spectrum is predicted just above the current experimental limits [48].

The interesting prediction of MSSMRN is a small contribution to the muon anomalous magnetic moment $g-2, \delta a_{\mu}$ :

$$
\delta a_{\mu}=3.537 \times 10^{-10} .
$$

With this contribution, the discrepancy of the theoretical value and the experimental one becomes within $3 \sigma$; i.e., our model satisfies a limit for $a_{\mu}$ at a 3 sigma level.

\section{B. Predictions for charged LFV}

Since the slepton mixing is induced by the existence of the Dirac-Yukawa couplings via the RGE effect, we have a sizable charged LFV (CLFV).

Figure 4 shows the branching ratio of LFV decays as a function of $M_{1}$ in three different cases. Current bounds (gray region) and future sensitivity (dashed line) are summarized in Table IV. All of the reaction rates are crudely proportional to the second lightest Majorana neutrino mass $M_{2}$. The dependence comes from the elements of the Dirac neutrino Yukawa matrix $\lambda_{\nu}$ that have large absolute values for a fixed active neutrino parameter $\left|\left(\lambda_{\nu}\right)_{i 2}\right| \propto M_{2}$. All curves satisfy the requirement to solve the ${ }^{7} \mathrm{Li}$ problem while the thick solid lines fulfill those for the ${ }^{7} \mathrm{Li}$ and ${ }^{6} \mathrm{Li}$ problems.

The parameter of the RH neutrino is narrowed down to a small space to solve the ${ }^{7} \mathrm{Li}$ and ${ }^{6} \mathrm{Li}$ problems and to generate successfully large lepton asymmetry. The predictions for $\operatorname{BR}(\mu \rightarrow e \gamma)$ and $\operatorname{BR}(\mu \rightarrow 3 e)$ lie in the range where the recent and near future experiment can probe. Our scenario can be precisely illuminated by combining LFV observables and unique collider signals [94-100]. It should be emphasized that when we consider the ${ }^{6} \mathrm{Li} /{ }^{7} \mathrm{Li}$ problems in the constrained MSSMRN, it is no surprise that we have

TABLE IV. Current bound and future sensitivity of branching ratio of LFV decays.

\begin{tabular}{lcc}
\hline \hline Process & Bound & Sensitivity \\
\hline$\mu \rightarrow e \gamma$ & $4.2 \times 10^{-13}[87]$ & $6 \times 10^{-14}[88]$ \\
$\mu \rightarrow 3 e$ & $1.0 \times 10^{-12}[89]$ & $1 \times 10^{-16}[90]$ \\
$\tau \rightarrow \mu \gamma$ & $4.4 \times 10^{-8}[91]$ & $1 \times 10^{-9}[92]$ \\
$\tau \rightarrow 3 \mu$ & $2.1 \times 10^{-8}[93]$ & $1 \times 10^{-9}[92]$ \\
\hline \hline
\end{tabular}


not yet observed CLFV. As a matter of fact, we will observe CLFV processes in the near future.

\section{SUMMARY AND DISCUSSION}

We have investigated the parameter space of the constrained minimal supersymmetric standard model with three RH Majorana neutrinos by requiring low-energy neutrino masses and mixings. At the same time, we have applied experimental constraints such as dark matter abundance, $\mathrm{Li}$ abundances, baryon asymmetry, and results from the LHC experiment, anomalous magnetic moment, and flavor observations. We have scanned the parameter of the complex orthogonal matrix $R$ in Eq. (10) assuming a relation among the RH neutrino masses (see Sec. IV B) and have found that the allowed parameter sets really exist where all of the phenomenological requirements are satisfied.

As shown in Sec. IV, the range of the lightest RH Majorana neutrino mass $M_{1}$ is roughly $10^{9} \mathrm{GeV} \leq M_{1} \leq 10^{11} \mathrm{GeV}$. The lower bound of $M_{1}$ is determined to obtain a sufficient amount of matter-antimatter asymmetry, while the upper bound is determined to suppress large lepton flavor violation. We have also found that the degenerate mass hierarchy of the active neutrinos is hardly realized in this region because rather large Yukawa couplings are necessary for the degenerate hierarchy. The flavor mixing among sleptons is significantly canceled through the renormalization group equation running by adjusting the complex angles. For this reason, the lightest slepton becomes a long-enough-lived particle, and we thus are able to solve the ${ }^{7} \mathrm{Li} /{ }^{6} \mathrm{Li}$ problems.

Furthermore, we have calculated the branching ratios of the lepton flavor violating decay using the allowed parameter sets. It is found that the upper bounds of $\operatorname{BR}(\mu \rightarrow e \gamma)$ and $\operatorname{BR}(\mu \rightarrow 3 e)$ are $\mathcal{O}\left(10^{-13}\right)$ and $\mathcal{O}\left(10^{-15}\right)$ for $M_{2}=$ $2 \times M_{1}$ and $\mathcal{O}\left(10^{-12}\right)$ and $\mathcal{O}\left(10^{-14}\right)$ for $M_{2}=4 \times M_{1}$, respectively. The LFV decays, $\mu \rightarrow e \gamma$ and $\mu \rightarrow 3 e$, are in the reach of MEG-II and Mu3e.

\section{ACKNOWLEDGMENTS}

This work is supported by JSPS KAKENHI Grants No. 25105009 (J.S.), No. 15K17654 (T. S.), No. 16K05325, and No. 16K17693 (M. Y.)
[1] P. F. de Salas, D. V. Forero, C. A. Ternes, M. Tortola, and J. W. F. Valle, arXiv:1708.01186.

[2] P. A. R. Ade et al. (Planck Collaboration), Astron. Astrophys. 594, A13 (2016).

[3] N. Aghanim et al. (Planck Collaboration), Astron. Astrophys. 596, A107 (2016).

[4] P. Minkowski, Phys. Lett. 67B, 421 (1977).

[5] T. Yanagida, Conf. Proc. C7902131, 95 (1979).

[6] M. Gell-Mann, P. Ramond, and R. Slansky, Conf. Proc. C790927, 315 (1979).

[7] S. L. Glashow, NATO Sci. Ser. B 61, 687 (1980).

[8] R. N. Mohapatra and G. Senjanovic, Phys. Rev. Lett. 44, 912 (1980).

[9] M. Fukugita and T. Yanagida, Phys. Lett. B 174, 45 (1986).

[10] V. A. Kuzmin, V. A. Rubakov, and M. E. Shaposhnikov, Phys. Lett. 155B, 36 (1985).

[11] J. A. Harvey and M. S. Turner, Phys. Rev. D 42, 3344 (1990).

[12] W. Buchmuller, P. Di Bari, and M. Plumacher, Nucl. Phys. B643, 367 (2002); B793, 362(E) (2008).

[13] J. R. Ellis and M. Raidal, Nucl. Phys. B643, 229 (2002).

[14] M. Bando, S. Kaneko, M. Obara, and M. Tanimoto, Prog. Theor. Phys. 112, 533 (2004).

[15] S. Chang, S. K. Kang, and K. Siyeon, Phys. Lett. B 597, 78 (2004).

[16] S. T. Petcov, W. Rodejohann, T. Shindou, and Y. Takanishi, Nucl. Phys. B739, 208 (2006).

[17] W.-l. Guo, Z.-z. Xing, and S. Zhou, Int. J. Mod. Phys. E 16, 1 (2007).
[18] S. Pascoli, S. T. Petcov, and A. Riotto, Nucl. Phys. B774, 1 (2007).

[19] D. N. Spergel et al. (WMAP Collaboration), Astrophys. J. Suppl. Ser. 170, 377 (2007).

[20] K. Griest and D. Seckel, Phys. Rev. D 43, 3191 (1991).

[21] S. Profumo, K. Sigurdson, P. Ullio, and M. Kamionkowski, Phys. Rev. D 71, 023518 (2005).

[22] A. V. Gladyshev, D. I. Kazakov, and M. G. Paucar, Mod. Phys. Lett. A 20, 3085 (2005).

[23] T. Jittoh, J. Sato, T. Shimomura, and M. Yamanaka, Phys. Rev. D 73, 055009 (2006); 87, 019901(E) (2013).

[24] R. H. Cyburt, B. D. Fields, and K. A. Olive, J. Cosmol. Astropart. Phys. 11 (2008) 012.

[25] L. Sbordone et al., Astron. Astrophys. 522, A26 (2010).

[26] A. Coc, S. Goriely, Y. Xu, M. Saimpert, and E. Vangioni, Astrophys. J. 744, 158 (2012).

[27] M. Asplund, D. L. Lambert, P. E. Nissen, F. Primas, and V. V. Smith, Astrophys. J. 644, 229 (2006).

[28] T. Kawabata et al., Phys. Rev. Lett. 118, 052701 (2017).

[29] T. Jittoh, K. Kohri, M. Koike, J. Sato, T. Shimomura, and M. Yamanaka, Phys. Rev. D 76, 125023 (2007).

[30] T. Jittoh, K. Kohri, M. Koike, J. Sato, T. Shimomura, and M. Yamanaka, Phys. Rev. D 78, 055007 (2008).

[31] M. Pospelov, Phys. Rev. Lett. 98, 231301 (2007).

[32] Y. Konishi, S. Ohta, J. Sato, T. Shimomura, K. Sugai, and M. Yamanaka, Phys. Rev. D 89, 075006 (2014).

[33] F. Borzumati and A. Masiero, Phys. Rev. Lett. 57, 961 (1986). 
[34] J. Hisano, T. Moroi, K. Tobe, and M. Yamaguchi, Phys. Rev. D 53, 2442 (1996).

[35] J. A. Casas and A. Ibarra, Nucl. Phys. B618, 171 (2001).

[36] J. R. Ellis, J. Hisano, S. Lola, and M. Raidal, Nucl. Phys. B621, 208 (2002).

[37] J. R. Ellis, J. Hisano, M. Raidal, and Y. Shimizu, Phys. Rev. D 66, 115013 (2002).

[38] S. Lavignac, I. Masina, and C. A. Savoy, Phys. Lett. B 520, 269 (2001).

[39] A. Kageyama, S. Kaneko, N. Shimoyama, and M. Tanimoto, Phys. Rev. D 65, 096010 (2002).

[40] F. Deppisch, H. Pas, A. Redelbach, R. Ruckl, and Y. Shimizu, Eur. Phys. J. C 28, 365 (2003).

[41] T. Blazek and S.F. King, Nucl. Phys. B662, 359 (2003).

[42] S. T. Petcov, S. Profumo, Y. Takanishi, and C. E. Yaguna, Nucl. Phys. B676, 453 (2004).

[43] B. Dutta and R. N. Mohapatra, Phys. Rev. D 68, 056006 (2003).

[44] J. I. Illana and M. Masip, Eur. Phys. J. C 35, 365 (2004).

[45] K. S. Babu, J. C. Pati, and P. Rastogi, Phys. Lett. B 621, 160 (2005).

[46] S. T. Petcov, T. Shindou, and Y. Takanishi, Nucl. Phys. B738, 219 (2006).

[47] L. Calibbi and G. Signorelli, Riv. Nuovo Cimento 41, 1 (2018).

[48] C. Patrignani et al. (Particle Data Group), Chin. Phys. C 40, 100001 (2016).

[49] S. Kaneko, J. Sato, T. Shimomura, O. Vives, and M. Yamanaka, Phys. Rev. D 87, 039904 (2013); 78, 116013 (2008).

[50] S. Kaneko, H. Saito, J. Sato, T. Shimomura, O. Vives, and M. Yamanaka, Phys. Rev. D 83, 115005 (2011).

[51] J. Edsjo and P. Gondolo, Phys. Rev. D 56, 1879 (1997).

[52] T. Nihei, L. Roszkowski, and R. Ruiz de Austri, J. High Energy Phys. 07 (2002) 024.

[53] K. Kohri, S. Ohta, J. Sato, T. Shimomura, and M. Yamanaka, Phys. Rev. D 86, 095024 (2012).

[54] T. Jittoh, K. Kohri, M. Koike, J. Sato, T. Shimomura, and M. Yamanaka, Phys. Rev. D 82, 115030 (2010).

[55] T. Jittoh, K. Kohri, M. Koike, J. Sato, K. Sugai, M. Yamanaka, and K. Yazaki, Phys. Rev. D 84, 035008 (2011).

[56] K. Kohri, M. Koike, Y. Konishi, S. Ohta, J. Sato, T. Shimomura, K. Sugai, and M. Yamanaka, Phys. Rev. D 90, 035003 (2014).

[57] M. Kusakabe, T. Kajino, T. Yoshida, and G. J. Mathews, Phys. Rev. D 81, 083521 (2010).

[58] R. H. Cyburt, J. Ellis, B. D. Fields, F. Luo, K. A. Olive, and V. C. Spanos, J. Cosmol. Astropart. Phys. 12 (2012) 037.

[59] M. Kusakabe, K. S. Kim, M.-K. Cheoun, T. Kajino, and Y. Kino, Phys. Rev. D 88, 063514 (2013); 88, 089904(E) (2013).

[60] M. Kusakabe, K. S. Kim, M.-K. Cheoun, T. Kajino, Y. Kino, and G. J. Mathews, Astrophys. J. Suppl. Ser. 214, 5 (2014).

[61] D. G. Yamazaki, M. Kusakabe, T. Kajino, G. J. Mathews, and M.-K. Cheoun, Phys. Rev. D 90, 023001 (2014).

[62] A. Coc, J.-P. Uzan, and E. Vangioni, J. Cosmol. Astropart. Phys. 10 (2014) 050.
[63] A. M. Mukhamedzhanov, Shubhchintak, and C. A. Bertulani, Phys. Rev. C 93, 045805 (2016).

[64] C. Bird, K. Koopmans, and M. Pospelov, Phys. Rev. D 78, 083010 (2008).

[65] K. Hamaguchi, T. Hatsuda, M. Kamimura, Y. Kino, and T. T. Yanagida, Phys. Lett. B 650, 268 (2007).

[66] M. Kamimura, Y. Kino, and E. Hiyama, Prog. Theor. Phys. 121, 1059 (2009).

[67] E. Nardi, Y. Nir, J. Racker, and E. Roulet, J. High Energy Phys. 01 (2006) 068.

[68] E. Nardi, Y. Nir, E. Roulet, and J. Racker, J. High Energy Phys. 01 (2006) 164.

[69] C. S. Fong, M. C. Gonzalez-Garcia, and E. Nardi, Int. J. Mod. Phys. A 26, 3491 (2011).

[70] T. Ishihara, N. Maekawa, M. Takegawa, and M. Yamanaka, J. High Energy Phys. 02 (2016) 108.

[71] D. J. H. Chung, B. Garbrecht, M. Ramsey-Musolf, and S. Tulin, J. High Energy Phys. 12 (2009) 067.

[72] C. S. Fong, M. C. Gonzalez-Garcia, E. Nardi, and J. Racker, J. Cosmol. Astropart. Phys. 12 (2010) 013.

[73] M. Plumacher, Nucl. Phys. B530, 207 (1998).

[74] L. Covi, E. Roulet, and F. Vissani, Phys. Lett. B 384, 169 (1996).

[75] M. Laine and M. E. Shaposhnikov, Phys. Rev. D 61, 117302 (2000).

[76] W. Porod, Comput. Phys. Commun. 153, 275 (2003).

[77] W. Porod and F. Staub, Comput. Phys. Commun. 183, 2458 (2012).

[78] G. Belanger, F. Boudjema, A. Pukhov, and A. Semenov, Comput. Phys. Commun. 180, 747 (2009).

[79] G. Belanger, F. Boudjema, P. Brun, A. Pukhov, S. RosierLees, P. Salati, and A. Semenov, Comput. Phys. Commun. 182, 842 (2011).

[80] D. Barducci, G. Belanger, J. Bernon, F. Boudjema, J. Da Silva, S. Kraml, U. Laa, and A. Pukhov, Comput. Phys. Commun. 222, 327 (2018).

[81] Y. Amhis et al. (HFLAV Collaboration), Eur. Phys. J. C 77, 895 (2017).

[82] V. Khachatryan et al. (LHCb, CMS Collaborations), Nature (London) 522, 68 (2015).

[83] D. Asner et al. (Heavy Flavor Averaging Group), arXiv: 1010.1589.

[84] G. W. Bennett et al. (Muon g-2 Collaboration), Phys. Rev. D 73, 072003 (2006).

[85] S. Davidson and A. Ibarra, Phys. Lett. B 535, 25 (2002).

[86] D. S. Akerib et al. (LUX Collaboration), Phys. Rev. Lett. 118, 021303 (2017).

[87] A. M. Baldini et al. (MEG Collaboration), Eur. Phys. J. C 76, 434 (2016).

[88] A. M. Baldini et al. (MEG II Collaboration), arXiv:1801 .04688 .

[89] U. Bellgardt et al. (SINDRUM Collaboration), Nucl. Phys. B299, 1 (1988).

[90] A. Blondel et al., arXiv:1301.6113.

[91] B. Aubert et al. (BABAR Collaboration), Phys. Rev. Lett. 104, 021802 (2010).

[92] T. Aushev et al., arXiv:1002.5012.

[93] K. Hayasaka et al., Phys. Lett. B 687, 139 (2010).

[94] S. Biswas and B. Mukhopadhyaya, Phys. Rev. D 79, 115009 (2009). 
[95] J. Heisig and J. Kersten, Phys. Rev. D 84, 115009 (2011).

[96] K. Hagiwara, T. Li, K. Mawatari, and J. Nakamura, Eur. Phys. J. C 73, 2489 (2013).

[97] M. Citron, J. Ellis, F. Luo, J. Marrouche, K. A. Olive, and K. J. de Vries, Phys. Rev. D 87, 036012 (2013).
[98] N. Desai, J. Ellis, F. Luo, and J. Marrouche, Phys. Rev. D 90, 055031 (2014).

[99] J. Heisig, A. Lessa, and L. Quertenmont, J. High Energy Phys. 12 (2015) 087.

[100] V. V. Khoze, A. D. Plascencia, and K. Sakurai, J. High Energy Phys. 06 (2017) 041. 